\title{
DESAFÍOS DE LA EDUCACIÓN Y RESPUESTA FORMATIVA EN LOS PROCESOS DE DES-INTEGRACIÓN SOCIAL A TRAVÉS DEL TRABAJO EN EL ÁMBITO DE LA INMIGRACIÓN EN ITALIA.
}

Resumen. El flujo migratorio registrado en Europa, en Italia posee propiedades particulares derivadas fundamentalmente por la posición geográfica de la frontera terrestre (en particular las regiones del noroeste) y su amplia frontera marítima del sur. Los extranjeros (regulares y/o clandestinos) una vez llegados a Italia intentan insertarse en el mercado laboral y normalmente consiguen un empleo en el ámbito de la agricultura, la construcción y la industria.

En este artículo se analizarán los factores que favorecen y/o frenan la inclusión de los grupos extranjeros en la sociedad italiana a partir de la participación en el mercado laboral, tomando como ejemplo la situación laboral de la región Puglia -para el sector de la agricultura- y la Toscana -para el sector de la industria-.

En esta reflexión se definen las particularidades de las actividades empresariales étnicas, que están caracterizadas por el aumento de la producción y los beneficios ecómicos en correspondencia con el empeoramiento de las condiciones de empleo y el nivel de vida de los grupos extranjeros trabajadores.

Palabras claves: inmigración, integración laboral y social, explotación de inmigrantes, actividades empresariales étnicas, trabajo de inmigrantes.

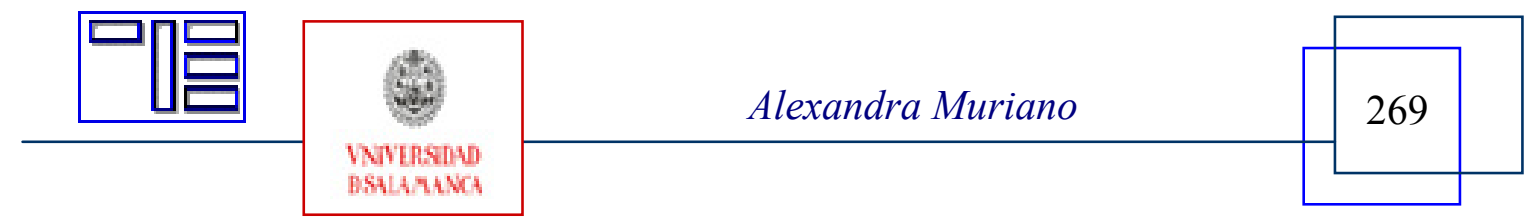


Revista Electrónica Teoría de la Educación.

Educación y Cultura en la Sociedad de la Información.

http://www.usal.es/teoriaeducacion

Vol. 9. No 2. Mayo 2008

\section{CHALLENGE OF EDUCATION AND FORMATIVE ANSWERS ON SOCIAL DISINTEGRATION PROCESS THROUGH WORK IN THE IMMIGRATION FIELD IN ITALY.}

Abstract. When examining European waves of migration, Italy is favored because of its terrestrial borders (particularly in the Northeast) and its maritime borders in the South. Legal and/or illegal immigrants arriving in Italy attempt to settle in the labor market and normally find work in agricultural areas, construction, and other industries accordingly. This article will investigate the factors that favor and/or slow down the integration of foreigners into Italian society beginning with their introduction into the workforce while examining the phenomena of social inclusion and exclusion as related to legal and/or illegal immigration in the industrial area of Tuscany and agricultural area of Apulia.

This article delineates patterns of labor activity in ethnic communities that are characterized by an increase in production and benefits in relation to the worsening of labor conditions and life of those affected.

Key words: Immigration, social integration, exploitation of immigrants, ethnic labor areas, immigrant employment, migrant work, migrant labor.

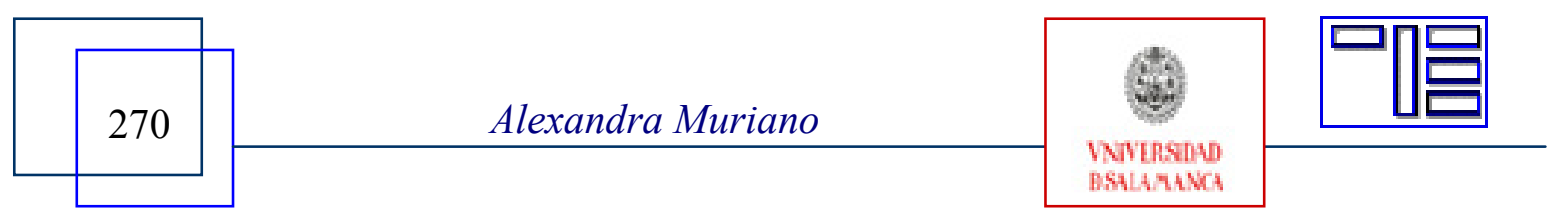




\section{DEFI DE L'ÉDUCATION ET RÉPONSES FORMATIVES CONCERNANT LES PROCESSUS DE DÉSINTÉGRATION SOCIALE À TRAVERS LE TRAVAIL DANS LE MILIEU DE L'IMMIGRATION EN ITALIE.}

Sommaire. Dans le contexte européen des flux migratoires, le cas italien est particulier en ce que les mouvements migratoires sont favorisés par la position géographique de frontière terrestre (en particulier les régions du Nord est) et de frontière maritime au sud.

Les étrangers (en règle et/ou clandestins) arrivent en Italie et cherchent à s'insérer dans le marché du travail. Normalement, ils trouvent un emploi dans les secteurs de l'agriculture, de la construction et de l'industrie.

Dans le présent article - qui distingue entre les phénomènes d'inclusion et d'exclusion sociale des travailleurs en règle, irréguliers et clandestins et prend pour exemple la situation du travail dans le secteur agricole dans les Pouilles et dans le secteur industriel en Toscane - nous procéderons à l'analyse des facteurs qui favorisent et/ou freinent l'insertion des étrangers dans la société italienne à partir de leur insertion dans le monde du travail.

Partant de cette analyse, nous pourrons esquisser des formes d'activité entrepreneuriale à base ethnique, caractérisées par une augmentation de la production et des bénéfices en corrélation avec une dégradation des conditions de travail des personnes concernées (i.e. les immigrés).

Mots- clés: immigration, intégration sociale et par le travail, exploitation des immigrés, secteurs de travail ethnique, emploi des immigrés.

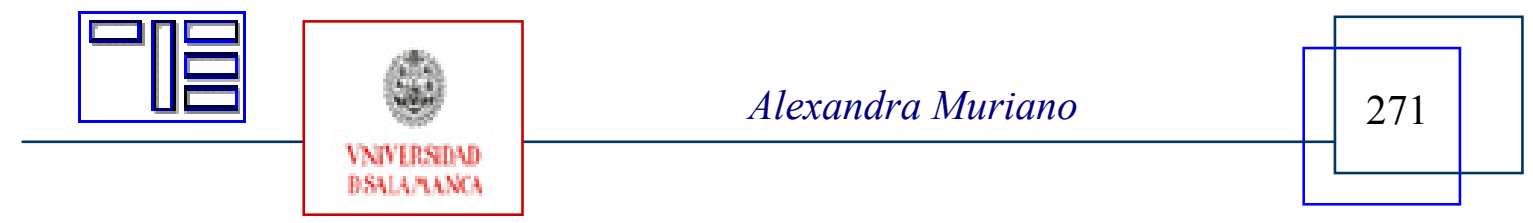


Revista Electrónica Teoría de la Educación.

Educación y Cultura en la Sociedad de la Información.

http://www.usal.es/teoriaeducacion

Vol. 9. No 2. Mayo 2008

\title{
DESAFÍOS DE LA EDUCACIÓN Y RESPUESTA FORMATIVA \\ EN LOS PROCESOS DE DES-INTEGRACIÓN SOCIAL A TRAVÉS DEL TRABAJO EN EL ÁMBITO DE LA INMIGRACIÓN EN ITALIA.
}

\author{
Alessandra Muriano \\ alessandra_muriano@yahoo.com \\ Universidad de Tübingen (Alemania).
}

\section{1.- INTRODUCCIÓN.}

En el presente trabajo se reflexiona sobre la inserción laboral de los inmigrantes en Italia, tratando de visualizar críticamente si esta participación en el trabajo resulta compatible con una inserción plena en la vida social italiana. Con este propósito se analizarán los mecanismos de participación instituidos dentro del mercado laboral italiano, tratando de establecer cómo favorecen o restringen una verdadera inserción social de los inmigrantes.

Para delinear estos factores se hará referencia a las investigaciones efectuadas en el territorio de manera particular relativamente a la mano de obra en las fábricas en Toscana y al empleo de trabajo temporal en la agricultura de Puglia, en todo caso sin omitir los fenómenos de explotación radical que se registran especialmente perjudicando a los segmentos de la población extranjera en el territorio nacional.

Utilizando los datos ISTAT y de investigaciones conducida por Médicos Sin Fronteras, para la región de la Toscana y por IRPET intentaré por lo tanto delinear un cuadro relativo al tipo de fenómeno de estudio y las conexiones entre la integración y la desintegración del inmigrante en el área socio-laboral.

En una segunda instancia nos concentraremos en la toma de posición que realiza la política educativa a través de sus diferentes programas en torno a promover los procesos de inclusión e integración de los grupos inmigrantes y asi también favorecer la aceptación de los ciudadanos autóctonos del fenómeno inmigratorio. En esta instancia nos abocaremos fundamentalmente a analizar los programas más relevantes de capacitación elaborados a nivel regional y con el apoyo de Ministerios Nacionales, para poder observar su impacto en la modificación de las posibilidades de inclusión social plena del colectivo inmigrante.

En el presente análisis se podrá observar las condiciones socioeconómicas y culturales heterogéneas así por ejemplo la condición de legalidad, el acceso a los servicios sanitarios y la situación de la vivienda, que inciden diferenciablemente en la inserción

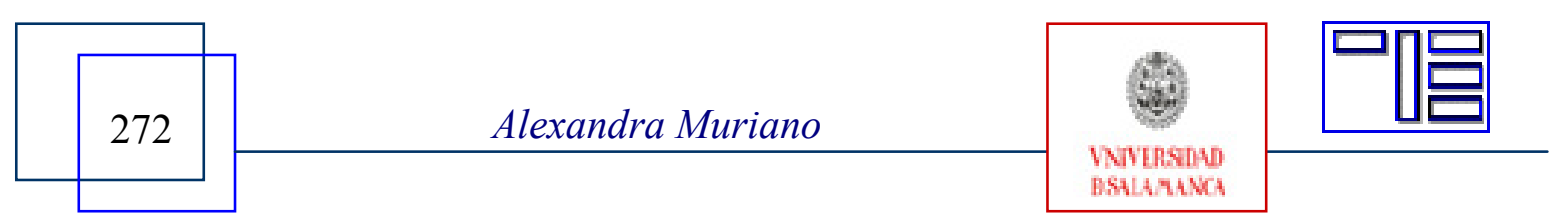


laboral y social de los inmigrantes, y que afectan la concretización de sus proyectos de vida personales.

A pesar de que el presente artículo se remitirá a reflexionar sobre factores que restringen la integración de los inmigrantes en la sociedad italiana, es de reconocerse aquí también que determinados factores, tendencias y lógicas de exclusión se hallan presentes en diferentes países europeos y del mundo, y, como se podrá apreciar finalmente el problema y los desafíos de la integración social plena de los inmigrantes, adopta un carácter global, que exige sin duda formas alternativas para repensar las vías de superación de este fenómeno cada vez más importante.

\section{2.- REFERENCIAS SOBRE LA COMPOSICIÓN Y LA DISTRIBUCIÓN DE LOS GRUPOS DE INMIGRANTES.}

La inmigración en Italia se introduce como un fenómeno fuertemente diferenciado en su interior. Un alto número de personas de varias nacionalidades, edades, clase social y nivel escolar se encuentra distribuido en el territorio de manera variada y peculiar al nivel regional; estas tendencias fueron modificando en el curso de los años, favoreciendo una diversificación constante de la distribución de las personas en el territorio.

Primeramente la diversificación de las presencias extranjeras en el territorio se debe por un lado a la posición geográfica de la frontera marítima al sur (por ej. en Sicilia se registra ya a partir de los años 70 la presencia preponderante de inmigrantes tunesinos por la cercanía geográfica y los bajos costos del recorrido), de la frontera terrestre de las regiones nordoriental (por ej. la cercanía entre Friuli Venezia Gulia y la ex Yugoslavia que abrieó un sistema migratorio con Europa oriental y los Balcanes) y de otro por los movimientos internos del norte al sur y al revés.

Posteriormente, las décadas de los 80 s y 90 s están caracterizadas por los nuevos y más complejos sistemas migratorios todavía hoy preponderantes, registrando un aumento de la presencia marroquí respecto a la tunesina ${ }^{1}$, de los inmigrantes provenientes de países asiáticos, como Filipinas, China, Sri Lanka, Bangladesh, y de los albaneses ${ }^{2}$.

Los datos ISTAT demuestran actualmente una incidencia preponderante de la presencia de tunecinos, albaneses y ex-yugoslavos. La isla de Sicilia sigue siendo tierra de desembarque para los tunecinos, Bari para los albaneses y Trieste para los Yugoslavos.

Con este cuadro breve es por lo tanto posible delinear una imagen de Italia caracterizada por varias presencias de inmigrantes, distribuidas de manera regional desigual.

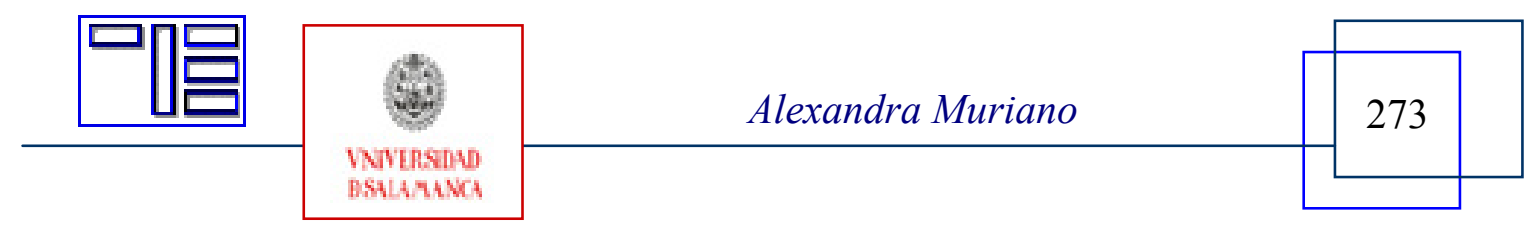


Revista Electrónica Teoría de la Educación.

Educación y Cultura en la Sociedad de la Información.

http://www.usal.es/teoriaeducacion

Vol. 9. No 2. Mayo 2008

\section{3.- ACTUAL DISTRIBUCIÓN DE INMIGRATES EN EL TERRITORIO.}

Como se ha precisado, la diversa proveniencia geográfica influencia de manera distinta al comienzo la colocación de los extranjeros en los estratos sociales del lugar de llegada.

Según el censo del 2001 es puede observar una distribución de la presencia de extranjeros en el territorio italiano muy alta en el norte y noreste (especialmente en Lombardía), seguida por las regiones del centro (especialmente en Lazio) y por último en las regiones del sur (particularmente Sicilia y Campania).

En 2002, al mismo tiempo de la ley "Bossi-Fini”, se registró un fuerte incremento de las autorizaciones - por lo tanto de las presencias de inmigrantes regulares para el trabajo temporal - pasando de 39.400 a 60.000 unidades y una contracción de las admisiones para el trabajo subordinado a tiempo indefinido y el trabajo independiente (disminuidos de 50.000 a 19.500) (Pastore, 2004).

Entre el verano y el otoño de 2002 se ha producido por lo tanto un cambio importante realizado por el procedimiento de la regularización de los trabajadores irregulares (más de 700.000 demandas), es decir la regularización de los trabajadores irregulares significa la disminución del trabajo negro.

Sin embargo, correspondientemiente con este proceso de regularización, se registró un aumento de las pérdidas de trabajo por los inmigrantes en el momento de la adquisición del permiso de estadía. Esta consecuencia no puede se considerada como una casualidad, al contrario como explica Pastore: "il passaggio ad uno stato di legalità permette l'acquisizione di diritti, uscendo dai contesti sociali di isolamento" (Pastore, 2004, 65), y en el caso de formas de trabajo negro implica la pérdida del trabajo.

La mayor presencia de inmigración residente en el 2004 se distribuyó así: Italia meridional recibió el $12 \%$, en el noroccidente de Italia se concentraba 36,3\%, seguida por la parte nororiental con el $27,2 \%$ y en la parte central del $24 \%{ }^{3}$.

La incidencia de la presencia extranjera en el total de la población residente era en el 2005 del 4,5\% con un incremento del 3,5\% con respecto al censo del 1993.

Las razones de esta distribución regional se pueden explicar a partir de dos factores que actuan como polos de atracción: por un lado los grandes centros urbanos y por el otro lado las áreas industriales. Efectivamente, en las grandes concentraciones urbanas se cuentan aproximadamente $1 / 3$ del total de la población extranjera, incidiendo en aproximadamente más del 4\% sobre la población local (sobretodo en Roma y Milano), mientras que en las provincias (Brescia, Treviso, Florencia) se trata de zonas con fuerte presencia de industrias. La distribución antes mencionada se desarrolla principalmente en dos líneas: la primera desde aquella de la llegada de inmigrantes y del movimiento

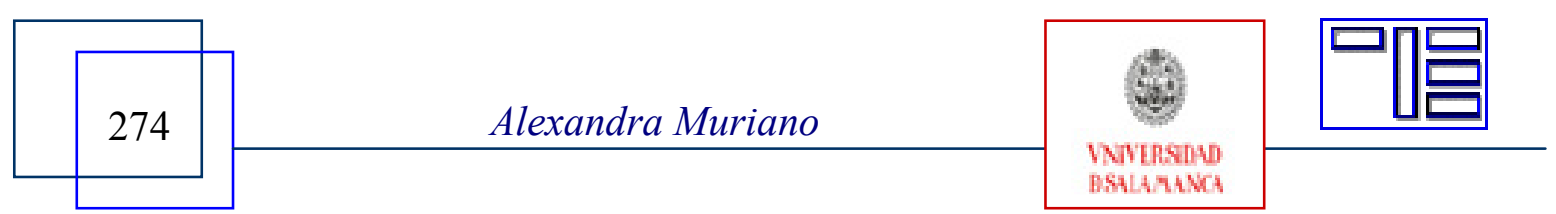


desde el sur hacia el norte; la segunda se refiere al movimiento desde los grandes centros hasta la provincia en la cual se sitúan los polos industriales.

Se observa una relación entre las áreas de destino y las oportunidades de trabajo.

\section{4.- LA POSIBILIDAD DE INSERCIÓN DE INMIGRANTES EN EL MUNDO DEL TRABAJO ENTRE LEYES, REALIDAD SOCIAL, MERCADO DE TRABAJO Y EL TRABAJO INFORMAL.}

\section{a) ¿Es el trabajo realizado por extranjeros complementario al de los italianos?}

Según muchos investigadores la mano de obra extranjera garantiza la demanda en áreas de trabajo poco atractivas, que los autóctonos por lo general se rehúsan a ocupar (por lo menos a iguales condiciones salariales), de tal manera que se sostiene la economía tradicional, por ejemplo: pesca, agricultura y ganadería (Pastore, 2004; Cardechi, Mottura, Pugliese, 2003; Bonifazi, 2007; Vizzini, 1983).

Según Brandolini:

"Le indicazioni delle varie evidenze microeconomiche (...) tendono a confermare l'interpretazione che vi sia stata finora complementarietà più che concorrenza tra la manodopera nazionale e immigrata (...). In un mercato del lavoro segmentato come quello italiano, in cui ampie sacche di disoccupazione coesistono con difficoltà nel reperimento della manodopera (...) $i$ lavoratori extracomunitari avrebbero coperto posti di lavoro di "bassa qualità", meno accetti alla manodopera italiana” (Brandolini, 2005, 261).

En mi opinión este análisis no tiene en cuenta dos factores: la diferencia del territorio y del desarrollo de las diferentes regiones y el nivel de la satisfacción del inmigrante respecto al trabajo realizado.

En el primer caso no se podría hablar de complementariedad en todo el territorio. En la parte del norte se registra una complementariedad por las razones explicadas por Brandolini, mientras en la parte meridional del país se registra una competencia directa o indirecta (Venturini, 1996; Bonifazio, 2003).

Cuando se habla de la competencia entre el inmigrante y el autóctono, se entiende que el trabajo del inmigrante favorece a la degradación de las condiciones de trabajo locales y/o impide su mejora (Reyneri, 1996), como sucede de hecho en los campos agrícolas meridionales en los cuales:

"Il consolidarsi di salari di fatto particolarmente bassi ha ridotto la disponibilità di alcune componenti giovanili dell'offerta di lavoro a occuparsi

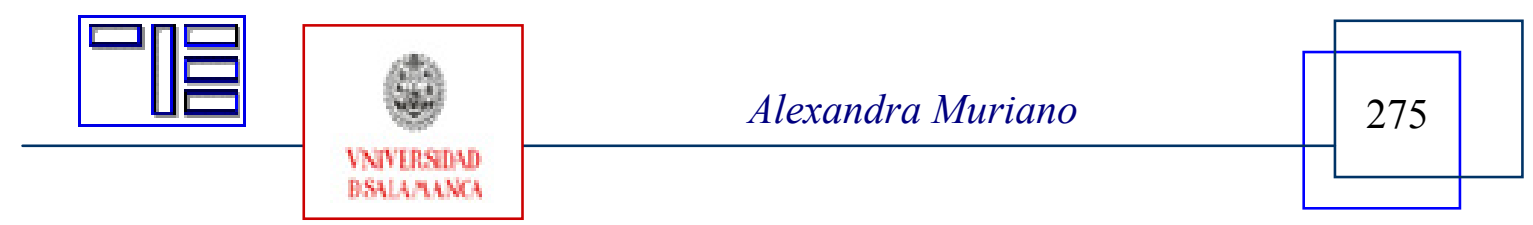


Revista Electrónica Teoría de la Educación.

Educación y Cultura en la Sociedad de la Información.

http://www.usal.es/teoriaeducacion

Vol. 9. $N^{\circ}$ 2. Mayo 2008

in agricoltura come dipendenti a tempo determinato, vista la situazione di ulteriore peggioramento delle condizioni di lavoro e soprattutto l'allontanarsi delle prospettive di cambiamento" (Macioti, Pugliese, 2003, 79).

En el segundo caso cabe preguntarse, si en el caso de que el inmigrante no se encontrase en la condición ya citada de aislamiento socio-legal aceptaría el empleo en áreas de trabajo "de baja calidad" y extremadamente flexible, lo cual asegura, según algunos, la complementariedad entre el trabajo autóctono y extranjero.

B) Inserción laboral y división en las áreas de trabajo en diferentes sectores.

A través datos ISTAT de la siguiente tabla es posible observar las modificaciones de los valores relativos a la inserción laboral de los inmigrantes en un intervalo de dos años, es decir 2001-2005, tomando en cuenta la distribución regional.

Gráfico 1. División áreas de trabajo de los inmigrantes empleados entre el 2001-2005.

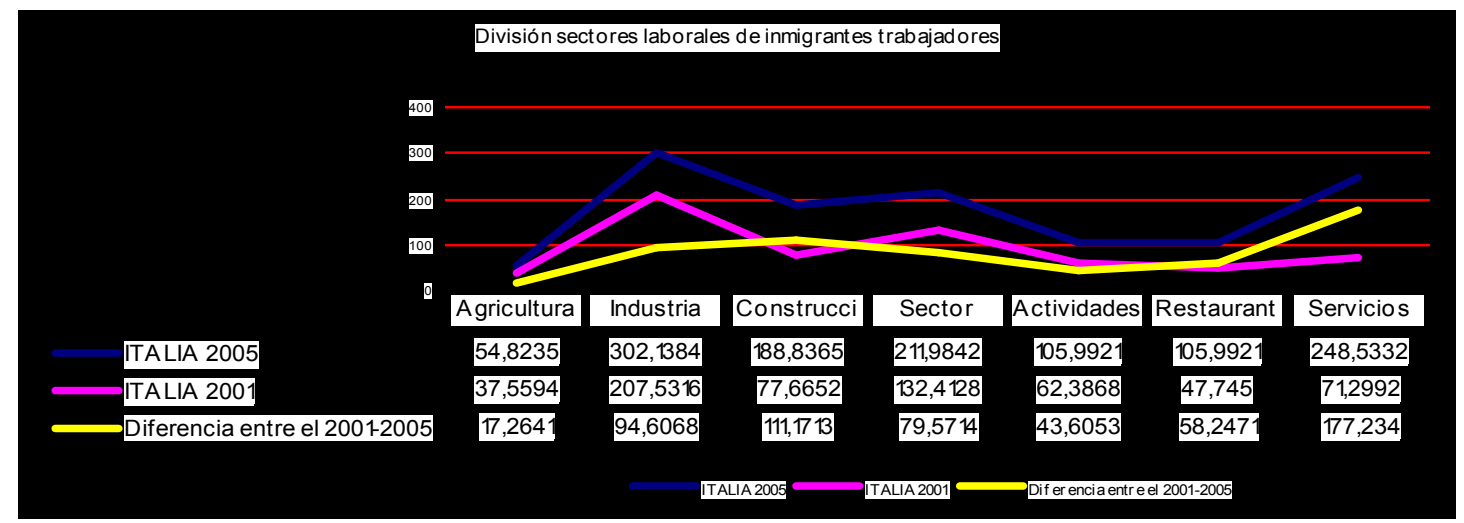

Fuente: Bonifazi, 2007, p.162-163; en base a datos de ISTAT

En base a una investigación conducida sobre la inmigración extranjera y la fuerza laboral en Italia, Bonifazi afirma que

"la crescita dell'occupazione straniera nell'industria (..) ha rappresentato un elemento estremamente importante nell'evoluzione del fenomeno. In molte aree dell'Italia centro-settentrionale di diffusa industrializzazione la dinamica economica ha da tempo sostanzialmente esaurito le riserve locali di lavoro, limitate anche per effetto delle tendenza demografiche in atto, spingendo le imprese a utilizzare nei processi produttivi lavoratori immigrati. L'edilizia appare invece uno dei principali settori di inserimento su tutto il territorio nazionale e, soprattutto, si caratterizza come un comparto di primo ingresso nel mercato del lavoro, spesso a prescindere da qualificazioni professionali o dalla regolarità della presenza" (Bonifazi, 2007, 166).

En el sector terciario tenemos que destacar el desarrollo de actividad empresarial de

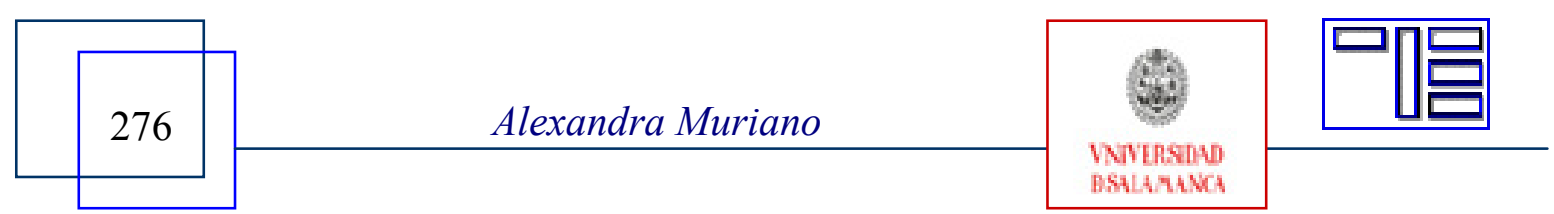


base étnica, de la cual los restaurantes, las tiendas y los laboratorios chinos representan la realidad más visible.

Las actividades agrícolas no se quedan atrás, por lo contrario, así lo demuestran los datos de ISTAT, representan el $14 \%$ en Italia meridional. Aquí de hecho los trabajadores inmigrantes son empleados en las operaciones de la recolección ${ }^{4}$, asegurando el desarrollo de las demás actividades agrícolas mediterránea.

c) La inserción de los inmigrantes: ¿un problema social o del mercado del trabajo?

Sería bueno considerar que si los procedimientos de regularización jurídica (sanatorie) han contibuido a la regularización de los inmigrantes, la adquisición del permiso de permanencia no siempre ha implicado también un trabajo de regularización, o en caso de un trabajo regularizado no ha implicado respecto de las condiciones exigidas a respecto del contrato nacional.

En unos casos apenas la adquisición del estado de "regular" ha decretado la pérdida del trabajo. Para abarcar este fenómeno tenemos que considerar que lo que hace interesante la mano de obra extranjera en el sector agrícola y en la construcción es por un lado la flexibilidad extrema, dictada por la necesidad de ingresos por parte de los inmigrantes, y por otro lado su debilidad contractual ${ }^{5}$. La posibilidad de emplearlos en negro, representa reducción gastos para el patrón.

Los datos recogidos por una investigación de un equipo de Médicos sin fronteras en el verano de 2004 en áreas rurales del sur del país en correspondencia a la temporada de la recolección han resultado que el $95,8 \%$ de los entrevistados empledos en la agricultura, no tenía un contrato de trabajo.

Estos datos atestiguan como tanto en el área de construcción como en el agrícola los inmigrantes son los más interesados por el fenómeno de la irregularidad del trabajo.

"L'ultima rilevazione del Nucleo dei Carabinieri dell'Ispettorato del lavoro svela un quadro gravissimo: su un campione di 21.431 aziende indagate nel corso del 2002, il 55\% è risultato impiegare personale "in nero". (...) Su 12.350 lavoratori stranieri fatti oggetto di ispezione il $26 \%$ era impiegato a nero e il $19 \%$ era privo di un titolo di soggiorno valido. Lo scarto tra $i$ due valori indica molto chiaramente che la irregolarità della condizione lavorativa interessa, oltre agli irregolari, anche una fascia assai ampia di regolari" (Pastore, 2006, 66).

Según Pastore:

"Benchè il cittadino italiano impiegato "in nero" abbia un costo ridotto per l'imprenditore, egli rappresenta in ogni caso un rischio, ad esempio di avviare

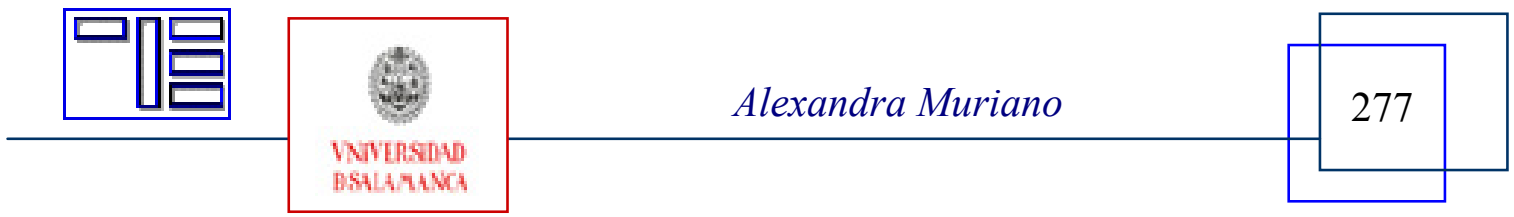


Revista Electrónica Teoría de la Educación.

Educación y Cultura en la Sociedad de la Información.

http://www.usal.es/teoriaeducacion

Vol. 9. No 2. Mayo 2008

una vertenza; nel caso dell'immigrato regolare i rischi per il datore di lavoro in caso di "lavoro nero" sono analoghi a quelli in cui si può incorrere avendo un lavoratore autoctono, proporzionalmente alla consapevolezza dei propri diritti e doveri, e livello di integrazione. Nel caso dello straniero privo di permesso di soggiorno, invece, questo rischio è remoto" (Pastore, 2006, 65)

Debemos tener presente que el hecho de trabajar en condiciones del irregularidad no coincide con el ser irregular o clandestino desde el punto de vista del permiso de permanencia, también porque hasta hoy la mayoría de los trabajadores en negro se componen de italianos.

Eso quiere decir que cuando se habla sobre la irregularidad uno se refiere sobre todo a la irregularidad contractual, la que representa los segmentos de la población expuestos a corto o a largo plazo, sobre todo en el momento de la llegada, a los mecanismos de la marginalización y vulnerabilidad socio-económica, particularmente atados al aislamiento y a la carencia del conocimiento de la lengua (para poderse informarse), de sus propios derechos y de las leyes que reglamentan el trabajo.

\section{5.- DOS CASOS DISTINTOS: LA INSERCIÓN SOCIO-LABORAL EN TOSCANA Y PUGLIA.}

El objetivo de este párrafo será de presentar dos realidades de inserción socio-laboral en dos regiones y en dos campos de empleo diferentes (agricultura e industria) ${ }^{6}$. Más que una comparación entre las dos regiones se trata de un análisis de los varios factores peculiares locales que contribuyen al vario grado de inserción en el tejido socio-laboral.

\subsection{La inserción en agricultura (Puglia).}

Según lo precisado, las áreas rurales siguen siendo unas de las áreas de actividades económicas donde se ha utilizado más el trabajo de los estranjeros.

En este párrafo se analizará también otra gran parte de los inmigrantes empleados en las campañas, es decir los solicitantes de asilo, refirendose a una investigación conducida por los Médicos Sin Fronteras en 2003 ${ }^{7}$.

En Puglia y en Basilicata se registra un porcentaje importante de extranjeros en posesión de un permiso de permanencia por solicitantes de asilo.

En 2003 se contaban 1400 solicitantes de asilo empleados en agricultura. Para explicar esta tendencia es necesario recordar que los solicitantes de asilo primeramente se encuentran recogidos en centros de identificación (en Puglia hay cuatro), los cuales al

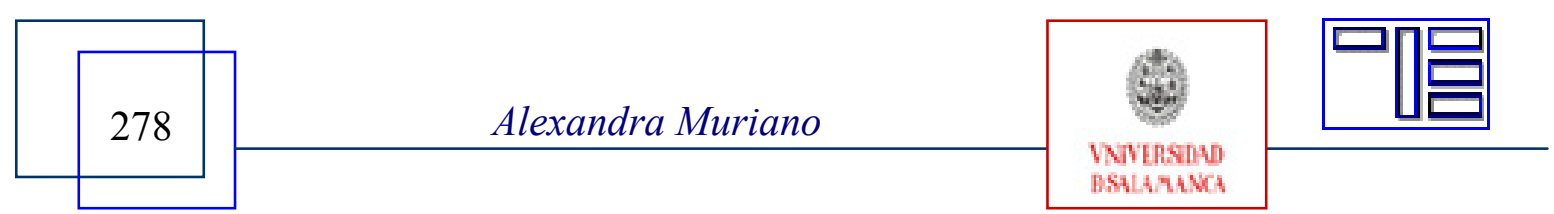


salir del centro y ante la falta de sustento económico, encuentran en la agricultura una oportunidad para ganar dinero para la supervivencia.

Siempre según los datos recogidos durante la investigación conducida por MSF resultó que el $97,5 \%$ han declarado ser trabajadores temporales, empleados en agricultura. El $100 \%$ de ellos declararon de carecer en un contrato de trabajo.

Es importante señalar que el elemento más significativo del sistema laboral en Puglia sigue siendo el fenómeno del caporalazo con el cual se recluta fuerza laboral. La incidencia del caporalazo en la remuneración resulta increiblemente onerosa.

La inclusión social de los inmigrantes empleados en las campañas no se beneficia realmente ni por la baja remuneración ni por el fenómeno del caporalazo. La falta de sueldos adecuados, o mínimos según el umbral de la pobreza establecido del estado italiano, no permiten la inserción del inmigrante empleado rural en viviendas "habitables". A esto va agregado el aislamiento del inmigrante causado por la carencia de conocimiento, a menudo, de la lengua y de sus derechos en el país, por lo cual se registra un alto procentaje de falta de acceso a los servicios médicos con las consiguientes patologías (respiratoria, dermatológica y gastroenterica) causadas por la pobreza.

El factor caractéristico del empleo de trabajadores extranjeros en agricultura es por cierto la temporaneidad de las ocupaciones, las cuales asociadas a la dificultad objetiva de encontrar una ocupación estable hace más difícil cualquiera proceso de ascenso o de inserción social.

\subsection{La inserción en el sector textil en el área de Prato (Toscana).}

La Toscana se encuentra entre las primeras cinco regiones italianas que cuentan una mayor presencia de extranjeros en el territorio; la incidencia de los residentes extranjeros regulares en la población total, coloca la región sobre del medio nacional que cuenta el 4,6\%.

La relación entre los flujos migratorios y el mercado de trabajo regional se caracteriza por el incremento sensible de la demanda de trabajo inmigrantes por parte de las empresas de Toscana y por un aumento del número de los ocupados extranjeros, acentuado particularmente unas tipologías laborales normalmente de bajo nivel y en particular en sectores tales como servicios sociales, manufacturas y construcción.

Otra particularidad de la región está representada por el desarrollo de formas de actividades empresariales étnicas, cuyo caso más emblemático está representado por la presencia china en el distrito industrial de Prato.

En este contexto se realizó en el 2006 una investigación por parte del IRPET y de la

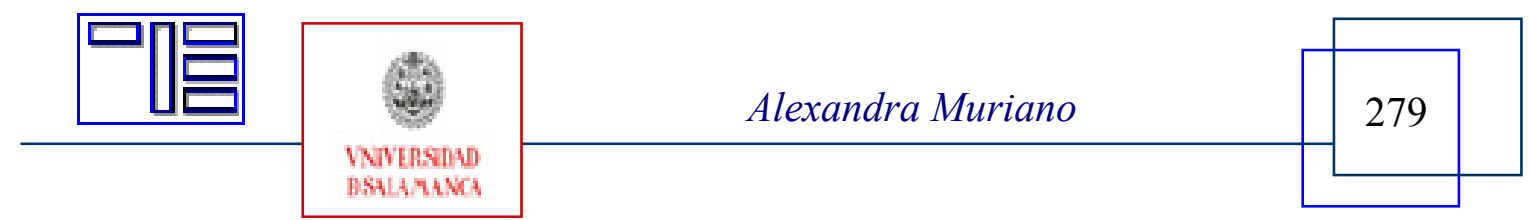


Revista Electrónica Teoría de la Educación.

Educación y Cultura en la Sociedad de la Información.

http://www.usal.es/teoriaeducacion

Vol. 9. No 2. Mayo 2008

región Toscana para analizar el fenómeno migratorio en relación a las condiciones de vidas y trabajo de los trabajadores extranjeros en tres ciudades de Toscana.

En el presente artículo se hará referencia a estos datos para reconstruir la relación entre las condiciones de vida y de trabajo, para poder entender cómo está relación influye en el proceso de integración social.

Los inmigrantes implicados en la investigación se encuentran ocupados en el sector textil y en el sector de la contrucción, como trabajadores genéricos, con tareas poco calificadas, que implican elevados factores de trabajo duro, esfuerzo y desazón (Carchedi, 2003; Pastore, 2004).

La necesidad de ganar dinero, las dificultades de comunicación, la carencia de la familia, la falta de relaciones sociales son algunos de los factores que favorecen, por lo menos por un período, la aceptación de trabajos que implican horarios onerosos.

A pesar de la posibilidad de inserción laboral, se registra malestar al exterior del tejido laboral, en relación al grado de integración con la sociedad y dificultades en encontrar una vivienda.

En cuanto a la inserción del inmigrante en el tejido social la Toscana se diferencia de las otras regiones por varios factores, sobre todo en relación a las oportunidades formativas. Prato se confirma como una zona por excelencia en donde está más difundido el conocimiento de la norma legislativa (con el 69\% valor más alto en toda la Toscana) y mayores oportunidades formativas sobre la seguridad en el trabajo (con un valor más alto del $71 \%$ ) y conocimiento de los servicios de asistencia social (64\%).

\section{6.- LA RESPUESTA EDUCATIVA COMO PRINCIPIO DE INTEGRACIÓN SOCIAL Y PROFESIONAL DE LOS INMIGRANTES. CAMINOS RECORRIDOS Y DESAFÍOS PARA EL FUTURO.}

Permitir la inclusión y la integración de los inmigrantes en la sociedad italiana implica prepararse para recibirlos. En la elaboración de soluciones de emergencia y coyunturales, o bien la exacerbación del trabajo como única vía para determinar la integración de los inmigrantes, se pueden evidenciar los mayores fracasos de la actual política de integración. En este sentido, resulta claro que pese a la existencia de acciones y programas diseñados para mejorar las ofertas y las condiciones laborales de los inmigrantes, estas medidas resultan insatisfactorias e incompletas al momento de acompañar los procesos educativos de formación educativa y adaptación cultural.

Teniendo en cuenta que los inmigrantes en la mayoría de los casos llegan a Europa por motivos de índole económico más que educativo y cultural (Bourdieu, 1998), la propuesta de un proyecto de formación de los inmigrantes, contenida en este estudio, no

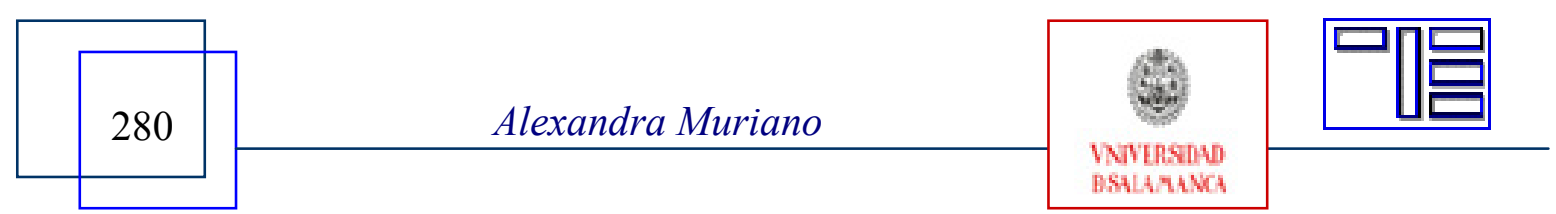


se refiere tanto a la formación primaria o escolar, sino a la promoción del individuo con respecto a las habilidades y a los conocimientos adicionales que se podrán adquirir en Italia, con el fin de mejorar su inserción en el entretejido social.

Las intervenciones políticas anteriormente esbozadas muestran una cierta debilidad en el debate político sobre las cuestiones de la educación y formación.

Si por un lado es evidente una subestimación de las necesidades educativas y culturales de los inmigrantes, por otro se diagnostica una falta total de reconocimiento de las calificaciones y de las experiencias profesionales adquiridas en los países de origen (y la consiguiente inclusión en sectores laborales para las cuales no se requiere formación especial), así como las dificultades de acceso a las actividades de formación (ya exigua) en favor de los inmigrantes, sobre todo cuando el acceso a la formación, la cual está permitida sólo a los que se encuentran en una situación regular $^{8}$ (con exclusión de los inmigrantes ilegales y refugiados, es decir, de los que se encuentran en condiciones precarias), y a los menores no acompañados y a las mujeres víctimas de la trata de prostitución ${ }^{9}$.

\subsection{El nivel de instrucción y los procesos de descalificación profesional.}

Para destacar brevemente los puntos anteriores, es útil mencionar algunas cifras del ISTAT, que muestran un grado alto de formación de los extranjeros. Los valores no justifican la inclusión de los inmigrantes en sectores laborales no correspondientes a sus títulos.

Gráfico 2. al nivel educativo de los inmigrantes frente a los italianos

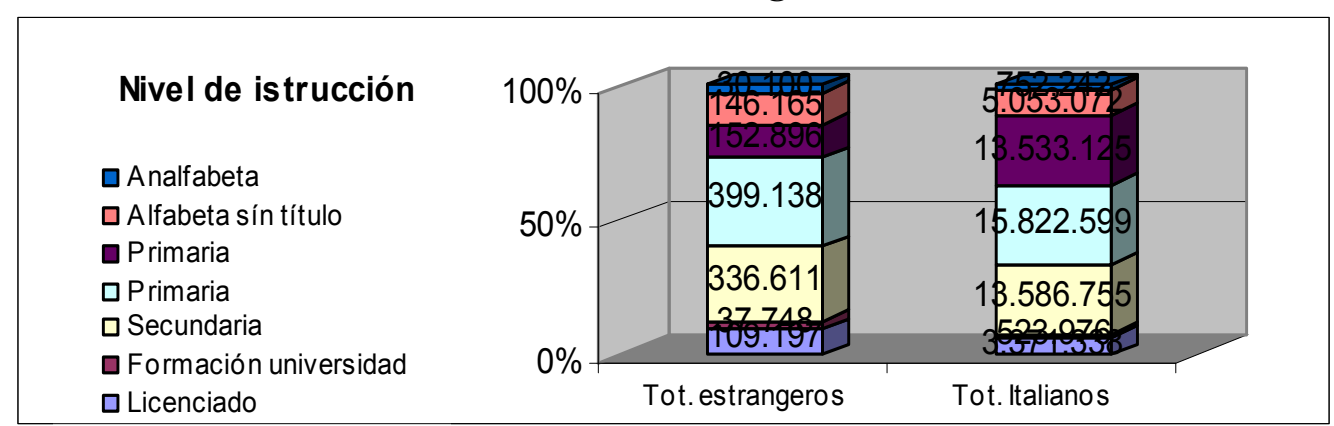

Fuente: ISTAT, por el 2001 datos online del " $14^{\circ}$ Censimento generale della popolazione e delle abitazioni", www.istat.it/istat/eventi/stranieri/volume_stranieri.pdf

No sólo por los datos Istat, sino también por los datos estadísticos de INPS y del Dossier Caritas, se evidencia la tendencia de un proceso de descalificación profesional, es decir, la contratación de inmigrantes en puestos de baja calificación en contradicción con los buenos niveles de educación que muchos de ellos poseen y con la experiencia profesional acumulada a lo largo de los años en Italia. El investigador del Dossier Caritas-Migrantes, Franco Pittau, dice que incluso un licenciado, si es inmigrante, en Italia se encuentra a menudo trabajando como obrero. Un tercio de los empleados

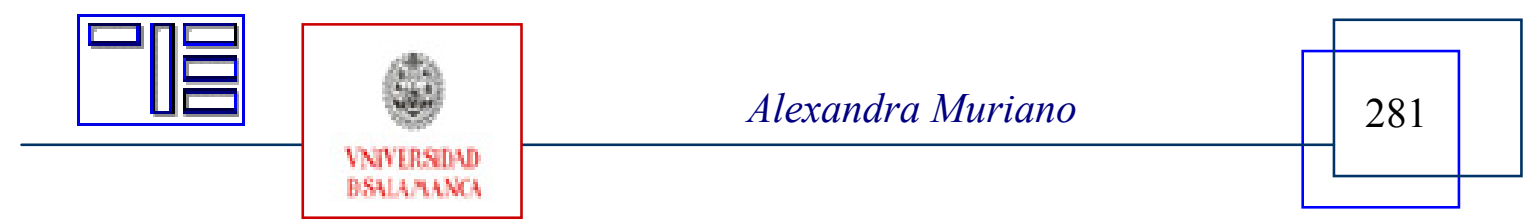


Revista Electrónica Teoría de la Educación.

Educación y Cultura en la Sociedad de la Información.

http://www.usal.es/teoriaeducacion

Vol. 9. No 2. Mayo 2008

extranjeros es de hecho (en comparación con el 10\% de los trabajadores en general) ubicado en el segmento más bajo del mundo del trabajo, realizando trabajos de baja calificación, por lo cual se requiere en la mayoría de los casos fuerza física y resistencia, es decir, un trabajo no calificado o una actividad de carácter manual.

\subsection{Consecuencias positivas de una política de formación de los trabajadores inmigrantes.}

Resulta claro que una orientación encaminada a la realización de ofertas de formación necesariamente tiene que ir más allá de una visión ideológica que solo atienda a la urgencia y la inmediatez de los problemas que afligen a los inmigrantes.

Sonetti (2006) en un ensayo sobre la formación de los trabajadores inmigrantes "Uomini senza qualità", observa en la formación que tiene el objetivo de preparación para el trabajo (formación en una perspectiva productiva, es decir, una inversión que tiende a mejorar la colocación de los trabajadores inmigrantes) y asi como también en la formación que se plantea el aprendizaje para la integración (inserción de obra inmigrante ya no pensada a priori según una lógica de persistencia en el de su propia subalternidad en el sistema social productivo) un papel importante para mejorar por un lado la imagen, que los que llegan pueden dar de sí mismos, y por otro lado la que los italianos tienen de ellos (desarrollando una cultura de iguales oportunidades).

En la práctica, también los comerciantes que utilizan el personal extranjero pretenden la formación para la integración laboral, en cuanto:

"los inmigrantes son vistos como personas "motivadas", dispuestas a aprender el trabajo, amigables y flexibles para el cliente. Las dificultades se refieren a la comprensión de la lengua, y un concepto diferente del "ritmo" y de la "higiene", factores más variables en los diferentes contextos culturales de pertenencia."

A partir del hecho que la formación de los inmigrantes tiene repercusiones en términos de empleo y de mejora de integración, me centraré en algunas de las actividades que se realizan con el fin de evidenciar sus aspectos positivos y sus limitaciones.

A nivel nacional, en relación con los instrumentos predispuestos por las empresas para promover la integración de los trabajadores extranjeros, los principales esfuerzos se centran en formas de apoyo por la búsqueda del hogar $(8,8 \%)$, reconocimiento de días festivos $(8,1 \%)$, servicios predispuestos por las empresas que tratan de facilitar la liberación de la documentación necesaria para obtener el permiso de estancia $(6,4 \%)$.

a) La respuesta de las instituciones en el área de la formación en Toscana y Puglia

En todo el territorio se observa una respuesta de las autoridades locales por la formación de los extranjeros. En Puglia sigue siendo limitada al aprendizaje del italiano como lengua extranjera, mientras que en Toscana se ha puesto especial énfasis en la educación

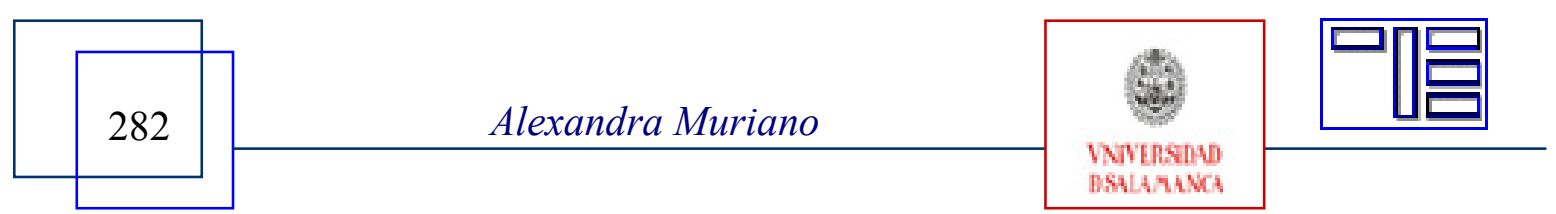


como vehículo para la integración, en donde la plenitud de derechos está garantizada cuando los trabajadores tengan las herramientas para un empleo calificado.

La red de educación de los adultos en la región Puglia se ocupa a través de CTP (Centros Territoriales Permanentes para la educación y la formación en edad adulta) de la formación de los extranjeros ${ }^{10}$ por un lado ofreciendo cursos de idioma italiano para extranjeros (disponible en $1 / 4$ de los CTP) por otro lado cursos para conseguir una calificación profesional (incidencia del 10\%), el total alrededor de 311 extranjeros por 3215 participantes $^{11}$.

La región de Toscana favorece el binomio "seguridad y derechos pasan a través de la formación en ámbito de la inmigración". Bajo los auspicios de este lema, organiza cursos de formación, enfocando la importancia de la educación, la cual representa para estos trabajadores uno de los principales vehículos de integración, que les permite adquirir, junto a los conocimientos del idioma, también conocimientos relativos a la seguridad en el lugar de trabajo y sus propios derechos, como el curso de capacitación sobre el papel y el funcionamiento de la autonomía local brindado en el 2005 por el Consiglio degli Stranieri della Provincia di Firenze y otras dos iniciativas: la educación a distancia a través de Internet (proyecto TRIO) y la formación en los países de origen ${ }^{12}$.

\section{7.- CONCLUSIÓN.}

En el cuadro que acabo de analizar surgen una serie de factores que caraterizan el fenómeno en análisis, entre los cuales tenemos que enumerar las tomas de decisiones por parte del estado en materia de migración ${ }^{13}$, la realidad económica italiana en el contexto nacional y global, el desempeño por parte de las política sociales y por último la respuesta educativa a esta problemática.

En relación a la realidad económica italiana en el contexto nacional y global, tenemos que observar que dentro del mercado global para apoyar la competición del mercado no es necesario el reclutamiento de grandes cantidades de fuerza laboral no calificada en el sector central de la producción, como sucedió antiguamente, sino de fuerza laboral sin capacidad contractual y altamente flexible, para asumirla al nivel periférico en los campos marginales de la producción.

Creo que es impensable tolerar la aceptación implícita de las condiciones de trabajo descritas, que llevan a un aumento de la producción y de los beneficios en correspondencia con un empeoramiento de las condiciones de trabajo y de vida. Se asiste a un proceso de un inferiorización de los trabajadores, es decir "lavoratori resi inferiori da un sistema economico, sociale e político" (Palidda S., 2000; Perrone, 2005).

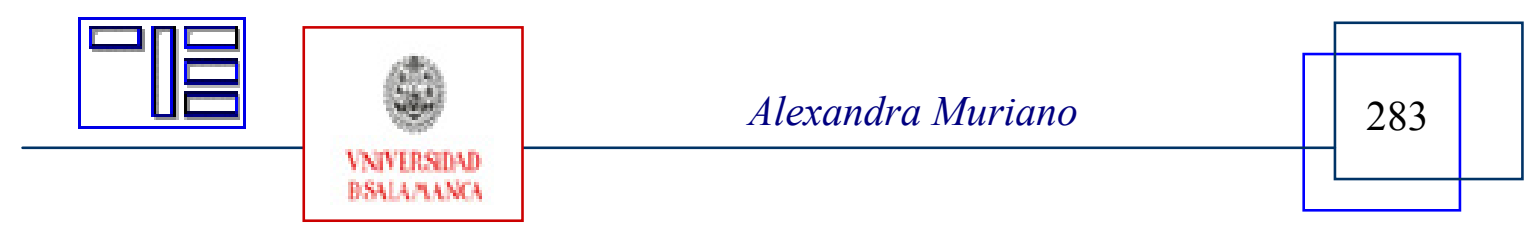


Revista Electrónica Teoría de la Educación.

Educación y Cultura en la Sociedad de la Información.

http://www.usal.es/teoriaeducacion

Vol. 9. No 2. Mayo 2008

Como precisado en los párrafos anteriores, se asiste por un lado a una absoluta falta de conocimiento de la ley por parte del inmigrante y por otro lado a una fuerte deficiencia de los servicios garantizados por parte del estado.

Por eso, los servicios sociales y los centros para el empleo deberían funcionar como distribuidor de información para el inmigrante con respecto a sus derechos en el país. Esto permitiría al inmigrante salir de su propia condición de fragilidad jurídica, factor determinante en el proceso de explotación laboral.

En razón las intervenciones producidas en el ámbito de la educación y la capacitación, se observa que a pesar de las múltiples acciones acometidas en todas las regiones, las empresas italianas en relación a las europeas, conceden una escasa atención a la formación de sus empleados inmigrantes: en muchos países de la Unión Europea casi 1 empresa de cada 5 organizan cursos de formación profesional para los extranjeros $(7,4 \%)$ o cursos de idiomas (12,3\%), en cambio en Italia sólo el 6,2\% de las empresas está trabajando en esta dirección.

Los programas y las políticas de intervención de apoyo a la integración de los inmigrantes actualmente en vigor, resulta conveniente profundizar el control y la evaluación de los mismos en las diferentes regiones ${ }^{14}$; para verificar si efectivamente estos esfuerzos estan conduciendo a la mejora de las condiciones de integración social.

Este control facilitará la planificación idónea de nuevas actividades que imbolucren a las escuelas y a las demandas de formación de los inmigrantes, de este modo se podrán superar la falta de contextualizacion y pertinencia que comportan algunos proyectos. En una verdadera conjunción de oferta y demanda se podran coquistar mejores prácticas

Como una alternativa a largo plazo que podría mejorar las condiciones de integración, se basa en una propuesta de formación para los inmigrantes de "largo alcance" que considere la competencia profesional detentada por los inmigrantes, garantice la adquisición de la lengua italiana (tanto en Italia como en el país de origen), apoye la adquisicion de los conocimientos vinculados a la seguridad en el trabajo y los derechos de los trabajadores, y amplíe la obtención de contratos de trabajo que estén a su vez articulados a procesos formativos.

Al tratarse de un programa integral que abarque y conjugue tanto el ámbito laboral como de formación educativa, se requiere inexorablmente de un consenso estratégico entre las empresas, la sociedad civil y el estado, puesto que sólo desde una alianza intersectorial será posible mejorar las condiciones de integración y participacion social de los inmigrantes en Italia y Europa.

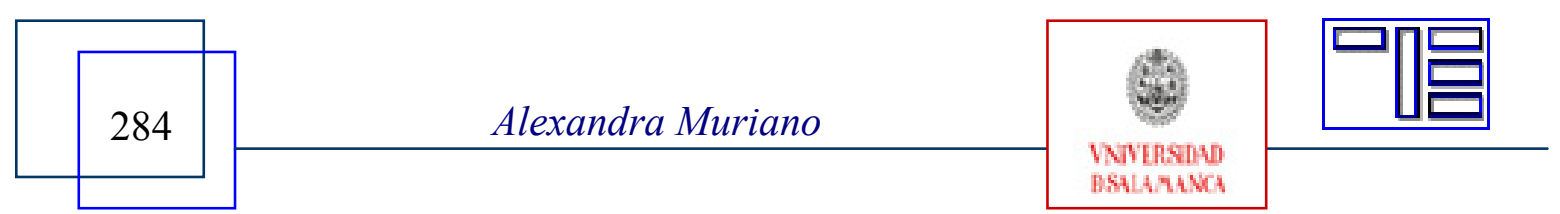




\section{8.- BIBLIOGRAFÍA.}

AMBROSINI, M. (2001). La fatica di integrarsi. Bologna: Il Mulino.

BRANDOLINI, A.; CIPOLLINE, P. y ROSOLIA, A. (2005). Le condizioni di lavoro degli immigrati in Italia. En LIVI BACCI (Edt.): L'incidenza economica dell'immigrazione, (pp.261-280). Torino: Giappichelli.

BONETTI, S. y FIORUCCI, M. (2006). Uomini senza qualità. La formazione dei lavoratori immigrati dalla negazione al riconoscimento. Milano: Ed. Guerini e Associati.

BONIFAZI, C. (2007). L'immigrazione straniera in Italia. Bologna: Ed. Il Mulino.

CAMPANI, G.; LAPOV, Z. y CARCHEDI, F. (2002). Le esperienze ignorate. Giovani migranti tra accoglienza, indifferenza e ostilità. Milano: Ed. Franco Angeli.

CARCHEDI, F.; MOTTURA, G. y PUGLIESE E. (2003). Il lavoro servile e le nuove schiavitù ( $2^{\mathrm{a}}$ edición). Milano: Ed. Franco Angeli.

CARCHEDI, F.; MOTTURA, G. Y PUGLIESE E (2004). Piccoli schiavi senza frontiere. Il traffico dei minori stranieri in Italia. Roma: Edizioni Ediesse.

COLOMBO, A.y SCIORTINO, G. (2004). Gli immigrati in Italia. Assimilati o esclusi: gli immigrati, gli italiani, le politiche. Bologna: Ed. Il Mulino.

CONFEDERAZIONE NAZIONALE DELL'ARTIGIANATO E DELLA PICCOLA E MEDIA IMPRESA. DOSSIER STATISTICO IMMIGRAZIONE/CARITAS DI ROMA (2003). Immigrati e imprenditorialità: realtà attuale e prospettive di sviluppo. Consultado el 7 de febrero de 2008 en: $<$ http://www.caritasroma.it/immigrazione/Dossier2003/Immigrati\%20e\%20Impre nditorialit\%E0\%202003.pdf>

CONSULENZA LEGALE.INFO (2008). Corsi di lingua italiana Associazione Ya basta, Bologna. Consultado el 7 de diciembre de 2008 en: $<\mathrm{http}: / /$ www.consulenza-

legale.info/index.php?option=com_content\&task=view\&id=538\&Itemid=80>

DOSSIER STATISTICO IMMIGRAZIONE, CARITAS/MIGRANTES (2002). Le rimesse: l'immigrato come operatore economico. Consultado el 7 de diciembre de 2008 en: <http://www.caritasroma.it/immigrazione/rimesse/rimesse.pdf>

FONDAZIONE ISMU INIZIATIVE E STUDI SULLA MULTIETNICITÀ (2003). VIII Rapporto sulle Migrazioni. Milano: Franco Angeli.

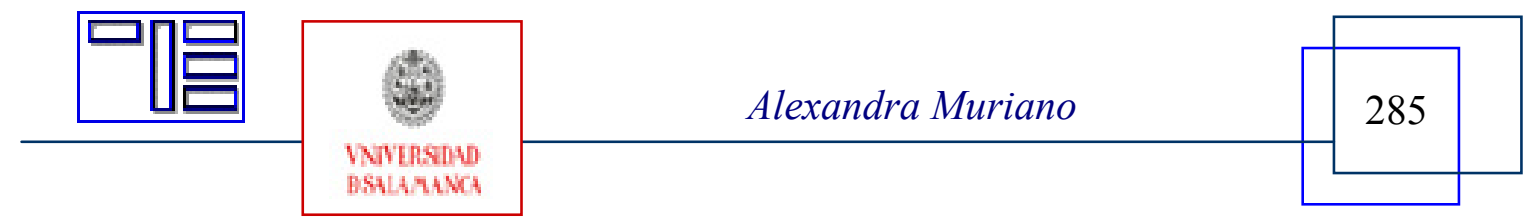


Revista Electrónica Teoría de la Educación.

Educación y Cultura en la Sociedad de la Información.

http://www.usal.es/teoriaeducacion

Vol. 9. No 2. Mayo 2008

GIOVANNI, F.; SAVINO, T. Y VALZANIA, A. (2006). La fabbrica dell'integrazione. Immigrati e industria diffusi in toscana. Collana lavoro - Studi e Ricerche/61. Pisa: ed. Plus.

ICSIM (ISTITUTO DI COOPERAZIONE E SVILUPPO ITALIA MOLDAVIA) (2003). Formazione e lavoro. Consultado el 12 de febrero de 2008 en: $<$ www.italiamoldavia.it/formazione $\% 20 \mathrm{e} \%$ 20lavoro.htm>

ISTAT (Istituto Nazionale di Statistica) (1993). La presenza straniera in Italia. Una prima analisi dei dati censuari. Roma: Istat.

ISTAT (Istituto Nazionale di Statistica) (1998). La presenza straniera in Italia negli anni '90. Roma: Istat.

ISTAT (Istituto Nazionale di Statistica) (2001). $14^{\circ}$ Censimento generale della popolazione e delle abitazioni, 100. Consultado el 25 de febrero de 2008 en: $<$ www.istat.it/istat/eventi/stranieri/volume_stranieri.pdf $>$

ISTAT (Istituto Nazionale di Statistica) (2006). La partecipazione al mercato del lavoro della popolazione straniera. I-IV trimestre 2005, "Statistiche in breve", 27 marzo.

ISTAT (Istituto Nazionale di Statistica) (2006b). Gli stranieri nella rivelazione sulle forze di lavoro, "Metodi e norme", 27.

ISTAT (Istituto Nazionale di Statistica) (2006c). Gli stranieri in Italia: analisi dei dati censuari. Roma: Istat.

ISTITUTO REGIONALE PROGRAMMAZIONE ECONOMICA TOSCANA (IRPET) (2006). La fabbrica dell'integrazione. Occupazione e sicurezza sul lavoro nell'industria diffusa. Consultado el 5 de abril de 2006 en:

$<$ www.irpet.it/storage/agendaallegato/102_Comunicato\%20stampa\%2028.2.pdf $>$

LABITALIA (2005). Toscana, formazione in patria per stranieri. Consultado el 16 de mayo del 2008 en: www.labitalia.com/articles/News/10746.html

MACIOTI, M.I. y PUGLIESE, E. (2003). L'esperienza migratoria. Immigrati e rifugiati in Italia. Roma-Bari: Laterza.

MELOTTI, U. (2004). Migrazioni internazionali. Globalizzazione e culture politiche. Milano: Bruno Mondatori.

MELOTTI, U. (1988). Dal Terzo Mondo in Italia. Studi e ricerche sulle immigrazioni straniere. Milano: Centro Studi Terzo Mondo.

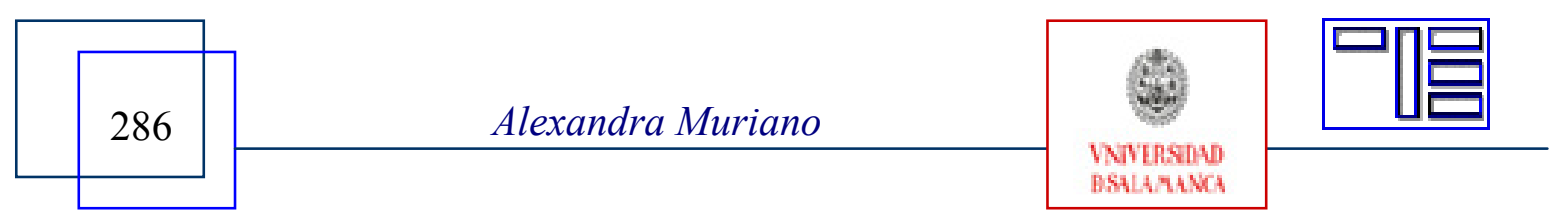


MINISTERO DELLA SOLIDARIETÀ SOCIALE (2007, 11 de octubre). "Decreto 16 luglio 2007. Fissazione del contingente di ingressi di cittadini stranieri per corsi di formazione professionale e tirocini formativi, per l'anno 2007," publicado en la Gazzetta Ufficiale (Diario Oficial de la Republica Italiana), Serie Generale (n.237, decreto del 16/07/2007). Consultado el 16 de mayo del 2008 en:

$<$ http://gazzette.comune.jesi.an.it/2007/237/3.htm>

MIUR (Ministero Italiano dell'Università e della Ricerca) - Ufficio Scolastico Regionale per la Puglia (2008). Seminario: l'educazione degli adulti in Puglia e prospettive. Consultado el 16 de mayo del 2008 en: $<$ http://www.puglia.istruzione.it/eda/eda.shtml $>$

MOFFA, C. (2002). La favola multietnica. Per una critica della sociologia dell'immigrazine facile. Torino: L'Harmattan Italia.

MSF -MEDICI SENZA FRONTIERE- (2005). I frutti dell'ipocrisia. Storie di chi l'agricoltura la fa. Di nascosto. Roma: Ed. Sinnos.

NOVE DA FIRENZE - GIORNALE ON LINE (2008). Corso di formazione per stranieri su ruolo e funzionamento degli enti locali. Consultado el 30 de marzo de 2008 en <http://www.nove.firenze.it/vediarticolo.asp? $i d=a 5.09 .16 .19 .10>$

OKKUPATI, M. (2008). Cna, in Italia circa 95 mila imprenditori stranieri. Consultado el 30 de marzo de 2008 en <http://www.okkupati.rai.it/news_lettura,1878.html>

PASTORE, F. (2004). Dobbiamo temere le migrazioni. Roma-Bari: Ed. Laterza.

PERRONE, L. (2005). Da straniero a clandestino. Napoli: Liguori Editore.

PALIDDA, S. (1994). Devianza e criminalità tra gli immigrati: ipotesi per una ricerca sociologica. En Inchiesta, 24, 103.

TOSCANA NOTIZIE. (2008). Immigrazione, sicurezza e diritti passano dalla formazione. Consultado el 22 de marzo de 2008 en: $<$ http://primapagina.regione.toscana.it/? codice $=15456>$

PROGETTO MELTINGPOT EUROPA (2008). Romania, altolá sulla frontiera occidentale. Consultado el 30 de marzo de 2008 en: $<$ http://www.meltingpot.org/articolo2774.html>

PUGLIESE, E. (2002). L'Italia tra migrazioni internazionali e migrazioni interne. Bologna: Ed. Il Mulino.

REYNERI, E. (1996). Sociologia del mercato del lavoro. Bologna: Il Mulino.

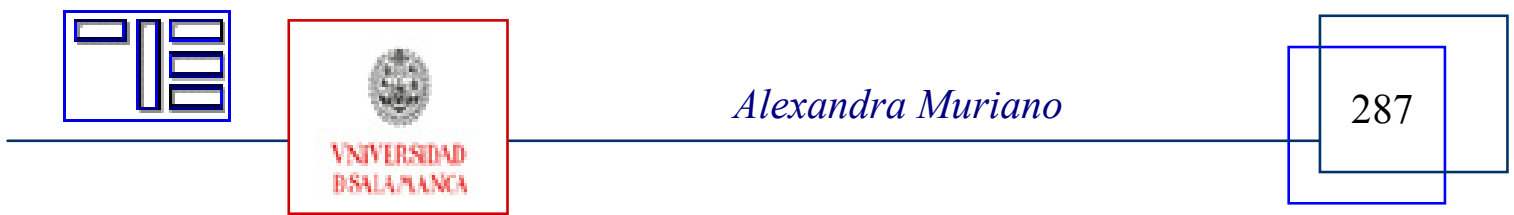


SONETTI, S. Y FIORUCCI, M. (2006). Uomini senza qualità, La formazione dei lavoratori immigrati dalla negazione al riconoscimento. Milano, Edizioni Angelo Guerini e Associati Stranieri in Italia.

STRANIERI IN ITALIA. IL PORTALE DEI NUOVI CITTADINI (2007). Bar $e$ ristoranti: il 25\% impiega stranieri. "Serve formazione". Gli esercenti chiedono più formazione professionale. Indagine Eurisko-Fipe". Consultado el 16 de julio del 2007

en: $<\mathrm{http}: / /$ www.stranieriinitalia.it/index.php?option=com_content\&task=view\&id=1 $581 \&$ Itemid $=151>$

STRANIERI IN ITALIA. IL PORTALE DEI NUOVI CITTADINI (2007b) ANSA: Immigrazione: a Milano un progetto salute per extracomunitari. Consultado el 16 de mayo del en $<$ http://www.stranieriinitalia.it/index.php?option=com_content\&task=view\&id=3 079\&Itemid=144>

STRANIERI IN ITALIA. IL PORTALE DEI NUOVI (2008): A Roma e Bologna, corsi gratuiti per migranti. Consultado el 30 de enero del 2008 en: $<\mathrm{http}$ //www.stranieriinitalia.it/index.php?option=com_content\&task=view\&id=2 481\&Itemid $=173>$

STRANIERI IN ITALIA. IL PORTALE DEI NUOVI (2008b): 4,4 milioni di euro per aiutare le vittime della tratta. Consultado el 28 de febrero del 2008 en: $<$ http://www.stranieriinitalia.it/index.php?option=com_content\&task=view\&id=2 786\&Itemid=17 3>

STRANIERI IN ITALIA. IL PORTALE DEI NUOVI (2008c): Progetto immigrazione sana , Comune di Milano. Consultado el 28 de marzo del 2008 en: $<\mathrm{http}: / /$ www.stranieriinitalia.it/index.php?option=com_content\&task=view\&id=3 079\&Itemid=144>

VENTURINI, A. (1996). Extent of competition between and complementarity among national and Third-world migrant workers in the labour market: An exploration of the Italian case. En L. FREY (Eds.): The Job and Effects of Migrant Workers in Italy. Three Essays, (pp.37 - 45). Geneva: ILO.

ZINCONE, G. (2000). Rapporto sull'integrazione degli immigrati in Italia. Bologna: Il Mulino.

ZINCONE, G. (2001). Rapporto sull'integrazione degli immigrati in Italia. Bologna: Il Mulino.
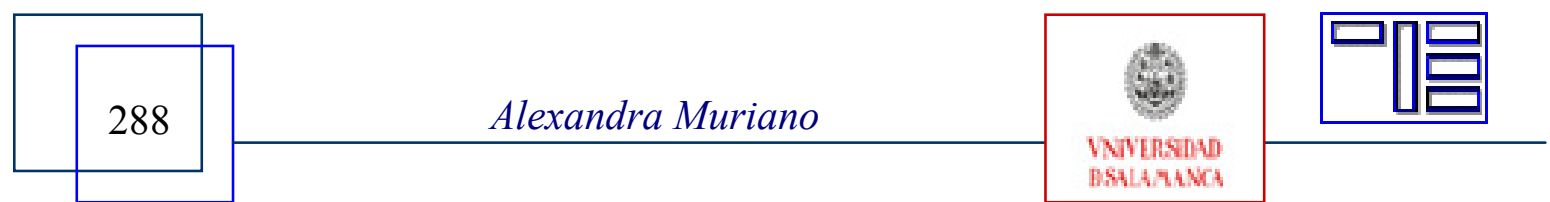


\section{Notas}

${ }^{1}$ La presencia de los marroquíes aumentó de aproximadamente unos pocos miles de personas al principio de los años '80s a 80 mil después de la legalización de inmigrantes del año 1990, manteniendo una presencia preponderante también en los años sucesivos, aproximadamente 2 de cada diez immigrates, contando en 2002 más de 170 mil presencias extranjeras (Colombo, Sciortino, 2004).

${ }^{2}$ Recordamos la llegada en masa de los albaneses y los dos desembarcos en Puglia del '91, más de 25.000 prófugos al primer desembarque posterior a la caída del régimen de Henver Hoxha, de hecho hoy Albania constituye la segunda nación en términos de presencias extranjeras. El mayor número de inmigrantes regular en 2002 proviene de los albaneses y de los tunesinos iguales al 11,4\% seguido por la nacionalidad de la Europa del este.

\section{${ }^{3}$ Fuentes otorgadas por ISTAT}

${ }^{4}$ La recolección exige la presencia de abundante mano de obra "temporal", en efecto se lleva a cabo en sólo un par de meses al año por región.

${ }^{5}$ Sin embargo tenemos que recordar que también aquellos que se encuentran sin documentos gozan del derecho fundamental de actuar en juicio (pero no reivindican antedicho derecho por miedo a ser identificados), mientras aquellos que se encuentran en una posición regular están ahora afuera de esa condición de aislamiento y de desventaje social o de conocimiento de sus prorpios derechos (Pastore, 2004).

${ }^{6}$ El análisis de la condición socio-laboral de los inmigrantes en Puglia parte directamente de una investigación operada por MSF en 2003 en la campaña de Puglia Basilicata con un grupo de trabajadores en la mayor parte de los casos irregulares; mientras que el análisis de la condición socio-laboral de la fuerza de trabajo extranjero en Toscana se refiere a las presencias de la población residente (segundo datos ISTAT) en el área de Prato, con el estado de la regulares.

${ }^{7}$ El plomo que examinaba de MSF ha interesado entre las varias áreas del sur la Puglia, particularmente el área de Foggia, y la Basilicata, limitadamente al área de Metaponto, cerca de la Puglia. El grupo de inmigrantes empleado en las campañas investigadas estaba compuesto del $90 \%$, de los hombres, de los cuales el $80 \%$ eran de area sub-sahariana y el $10 \%$ de las mujeres.

${ }^{8}$ La formación es tan importante para la integración social y cultural de los inmigrantes dentro de un plan de reglamentación y de programación de los flujos. Con el decreto legislativo (DL) 25.07.98, n. 286 del texto único de las disposiciones relativas a la reglamentación de la inmigración y las normas relativas a la condición de los extranjeros, el art. 27 reglamenta la entrada de personas autorizadas a permanecer por razones de formación profesional, desempeñando en períodos temporales formación en empresas italianas, realizando trabajo subordinado, es decir que los extranjeros pueden entrar a Italia para participar en capacitación y orientación en función de la terminación de una enseñanza profesional. Con decretos ministeriales (DM), sin embargo, las cuotas pueden acceder a la frecuencia de estos cursos. En el 2005 la cuota fue determinada por el Ministerio de Trabajo y Política Social, (5000 entradas para los extranjeros autorizados a asistir a los cursos y 5000 entradas para capacitación a una enseñanza), mientras que con el decreto de 16.06.07, el Ministerio de Solidaridad Social, determinó este flujo para el año 2007 confirmando 5 mil extranjeros, distribuidos entre las diferentes regiones, por lo cual están asignados a Apulia 175 escaños, mientras que la Toscana 370.

${ }^{9}$ Entre los proyectos para lo inmigrantes recordamos el curso de formación brindado este año 2008 en Roma para favorecer la inserción laboral de mujeres, a base étnica, que establece la participación "gratuita" de mujeres inmigrantes con "permiso de residencia", en busca del primer empleo y el curso "Operador de integración social y laboral multiétnico y multicultural", cuyo objetivo es la capacitación

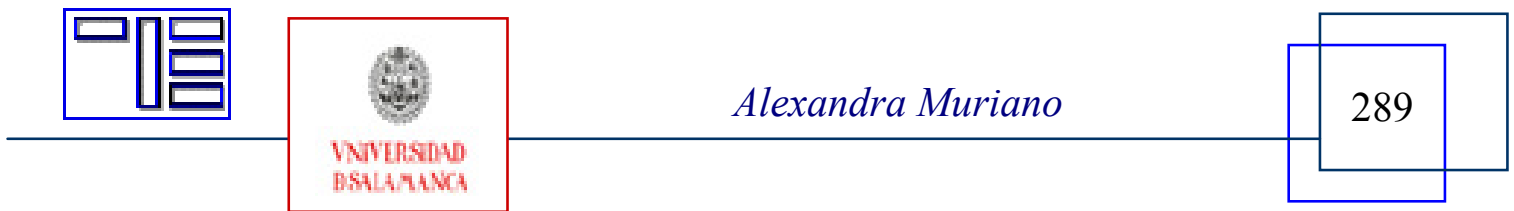


Revista Electrónica Teoría de la Educación.

Educación y Cultura en la Sociedad de la Información.

http://www.usal.es/teoriaeducacion

Vol. 9. No 2. Mayo 2008

deasistencia social utilizable en favor de los inmigrantes en los centros de acogida. Además, cursos gratuitos de italiano brindado por "Ya Basta!" Asociación de Bologna con el fin de facilitar la inclusión social y el empleo de los inmigrantes, con o sin permiso de residencia, y por fin el proyecto "Inmigración saludable", brindado por el municipio de Milán, en favor de las extranjeras para ofrecer un apoyo desde el punto de vista de la salud y la prevención en el área de Milán, en las zonas más expuestas a los fenómeno de droga y prostitución (y enfermedades relacionadas), utilizando un observatorio que vigile la salud de la población inmigrante y dos áreas de información en las zonas de mayor riesgo; en el año 2008 el Departamento de Igualdad de Oportunidades del Consejo de Presidencia financió 4.400,000 euros por proyectos de asistencia e integración promovidos por las asociaciones y las autoridades locales, para la recuperación y la integración de las víctimas de la explotación y el tráfico de prostitución.

${ }^{10}$ La realización de cursos ha sido garantizada en las instituciones educativas de la Región Puglia invirtiendo recursos "tavolo di emergenza" para promover la aceptación de los inmigrantes.

${ }^{11}$ Los datos se refieren a las actividades en el 2001, mientras que en el 2003 aún sin recursos específicos, los "CTF" (Centros Territoriales de Formación) han seguido la organización de cursos de lengua italiana como idioma extranjero y cursos de lengua madre.

${ }^{12}$ Se trata de un plan brindado por la región Toscana financiado con recursos ad hoc por el Ministerio de Trabajo y por la región (superior a 400.000 euros). Estos recursos realizarán la ejecución, en 3 países en desarrollo con intervenciones de formación específica antes de la salida. El plan se encuentra en conformidad con la ley Bossi-Fini, que contempla la concesión de licencias de entrada en Italia a los ciudadanos de terceros países que, en sus proprio países, hayan adquirido educación y formación finalizada a la inserción laboral.

${ }^{13}$ El estado define los límites de los permisos de permanencia permitidos en relación a los requisitos del mercado de trabajo italiano, la ley por lo tanto considera al inmigrante como mera fuerza de trabajo.

${ }^{14}$ Se refiere a las experiencias de formación realizadas por diversas razones, pues como se mencionó anteriormente, el Estado no guía la gestión directamente sino delega a través de financiamientos, las asociaciones locales a la prestación de servicios y cursos de capacitación para los inmigrantes.

Para citar este artículo puede utilizar la siguiente referencia:

MURIANO, Alessandra (2008). Desafíos de la educación y respuesta formativa en los procesos de des-integración social a través del trabajo en el ámbito de la inmigración en italia. En APARICIO, Pablo (Coord.) Desde la diversidad hacia la desigualdad: ¿destino inexorable de la globalización? [monográfico en línea]. Revista Electrónica Teoría de la Educación: Educación y Cultura en la Sociedad de la Información. Vol. 8, $\mathrm{n}^{\circ} 2$. Universidad de Salamanca. [Fecha de consulta: $\mathrm{dd} / \mathrm{mm} / \mathrm{aaaa}]$.

$<$ http://www.usal.es/ teoriaeducacion/rev_numero_09_02/n9_02_muriano.pdf $>$ ISSN 1138-9737

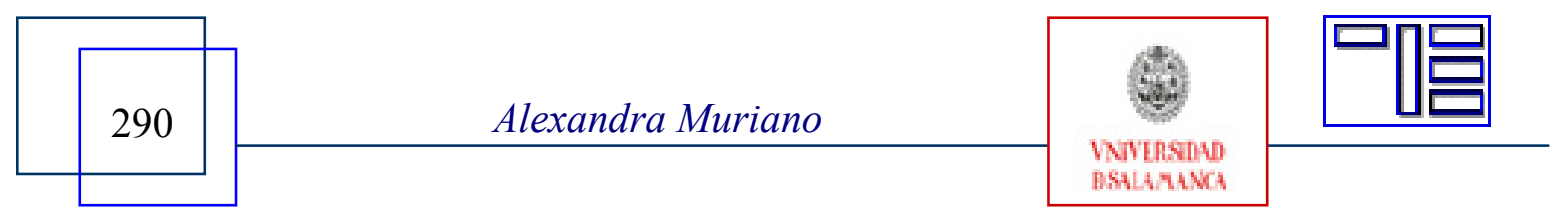

